Original Research Paper

\title{
Understanding Material Deformation in Nanoimprint of Gold using Molecular Dynamics Simulations
}

\author{
Abhaysinh Gaikwad and Salil Desai \\ Department of Industrial and Systems Engineering, \\ North Carolina Agricultural and Technical State University, Greensboro, USA
}

Article history

Received: 18-04-2018

Revised: $30-04-2018$

Accepted: 17-05-2018

Corresponding Author:

Salil Desai

Department of Industrial and

Systems Engineering, North

Carolina A and T State

University, Greensboro, USA

Email: sdesai@ncat.edu

\begin{abstract}
Material deformation in nanoimprinting is explored through Molecular Dynamics (MD) simulations. Understanding the behavior of substrate material during the nanoimprinting process is imperative for high resolution nanoscale pattern transfer. Nanoimprinting of the gold has the potential to make significant impact in diverse areas such as microelectronics and biomedical research. The deformation of gold during nanoimprinting is studied for varying depths of mold from the density profile mechanics viewpoint. The effects of varying imprint depth at a constant imprint velocity and temperature on the deformation behavior are investigated. It is observed that for higher imprint depth the spring back effect is substantial. Localized variations to density of the gold substrate were observed during the mold loading phases. Higher imprint depths displayed an increase of density up to 1.55 times the normal density of gold. This research provides insight into the deformation mechanism of gold substrate based on density profile variations and spring back effect.
\end{abstract}

Keywords: Density Profile, Imprint Depth, Molecular Dynamics, Nanoimprinting, Spring Back Effect

\section{Introduction}

The manufacturing of nanoscale structures is vital for the development of various types of functional nanodevices. Nanoimprint lithography (NIL) is a nanofabrication technique that facilitates fabrication of nanopatterns to produce integrated circuits, miniaturized devices, drug delivery nanoneedles (Chou et al.,1996; Chou and Krauss, 1997). Nanoimprint lithography is a versatile technology because of its high resolution, low cost and high throughput. In NIL, the precisely patterned mold is pressed into the substrate to replicate the mold topography. Nanoimprint lithography was originally developed for imprinting on polymers. However, direct nanoimprinting on metals is of great interest due to its potential applications (Pease and Chou, 2008). The micro/nanodevices made from metal hold advantages in applications such as gratings for optical components, polarizers, electromagnetic relays and optical isolators (Vigneswaran et al., 2014; Yang et al., 2014). Due to its superior electrical and thermal conductivity properties gold is an attractive candidate for the nanopatterning based on its widespread use in the electronic industry. Recently, gold has been used in the nanofabrication of medical sensors that detect biomolecules and diagnose specific diseases (Wong et al., 2013).
Nanoimprint lithography has been applied to fabricate devices for optical, photonics and many other applications in Micro Electro Mechanical Systems (MEMS) (Wu and Hou, 2018). Prior studies have provided insights on the effect of temperature on the imprint force for polymers (Kang et al., 2010). The atomic scale mechanism prompting the nanoimprint process for gold is unexplored. This includes investigating the density variation during material deformation and effect of imprint depth on the imprint quality. Specifically, the density variation during the plastic deformation of a metal during imprinting plays a vital role in determining the performance of the fabricated nanopatterns. Therefore, a fundamental understanding of the nanoimprint mechanism for gold is vital for fabricating high quality nanopatterns. Some researchers have explored direct nanoimprinting of gold with nanoindentation equipment. However, this work mainly focused on the experimental analysis (Zhang et al., 2014). Kwon and Kim (2016) presented the correlation between the temperature and the imprint force. They revealed that when temperature was increased the imprint force required was reduced. Although, experimental approaches explain outcomes of different imprint conditions, computational models provide a fundamental understanding of the process mechanics. Finite Element Method (FEM) has been 
applied to understand the phenomenon of nanoimprinting (Yasuda et al., 2010). However, the FEM is limited to the macro scale phenomenon and the continuum approach does not yield conclusive insights at the nanoscale regime (Cordeiro and Desai, 2016). Molecular dynamics modeling is an atomistic simulation to analyze the nanoscale phenomena (Cordeiro and Desai, 2015; 2016; 2017). Many researchers have focused their efforts on finding the optimal process parameters (Hsu et al. 2005), mold and pattern breakage mechanism (Yasuda et al., 2010) and effects of nanoscale defects on mold fracture (Lin and $\mathrm{Yu}, 2017$ ). A group of researchers have employed MD simulations to study the effects of system temperature, indentation force and surface orientation in the nanoindentation process to analyze the mechanical properties of the materials (Hsiung et al., 2009; Jiao et al., 2018). Quang-Cherng et al. (2004) investigated the effect of mold geometry on imprint forces and internal energies for nanoimprinting of copper. Based on load-distance and energy-time plots they concluded that the deformation force and the internal energy is high for greater taper angles. Also, the largest stress distribution was observed near the contact of mold and substrate.

This research investigates the material deformation behavior of gold substrate with a silicon mold. The effect of varying the imprint depth on the density variation in the gold substrate was studied. Understanding the density distribution within a nanoimprinted substrate is vital to the output quality of the reproduced pattern. This paper provides the fundamental understanding of the nanoimprint process with respect to metallic substrates wherein the spring back effect plays a dominant role in the final pattern transfer process.

\section{Methodology}

This section presents the simulation conditions, force fields and simulation analysis methods used. All the simulations were executed within Nanoscale Molecular Dynamics (NAMD) source code (Phillips et al., 2005). Visual Molecular Dynamics (VMD) was used for model development, visualization (Humphrey et al.,1996). Both NAMD and VMD were developed by the Theoretical and Computational Biophysics Group in Beckman Institute for Advanced Science and Technology at the University of Illinois at Urbana-Champaign. The simulations were run on a 64-bit Linux Fedora 27 system with Graphical Processing Unit (GPU) board ( 2 NVIDIA K40) with 2880 cores. The density profile tool plugin in VMD was used for analyzing the results of the simulations (Giorgino, 2014).

Figure 1 depicts the nanoimprint process for gold substrate and silicon mold. The nanoimprint process was divided into six steps which include system temperature ramp up, stamp loading into the substrate, stamp hold, system temperature ramp down, relaxation of the system and stamp unloading. Studying the dynamic behavior of substrate during these steps is important for successful pattern transfer.

In this study, the Lennard-Jones potential was used to simulate the atomic force interactions between mold and substrate which is defined by Equation (1):

$$
E_{L j}=4_{\varepsilon i j}\left[\left(\frac{\sigma_{i j}}{r_{i j}}\right)^{12}-\left(\frac{\sigma_{i j}}{r_{i j}}\right)^{6}\right]
$$

where, $E_{L j}$ signifies the pair potential energy function. The $\varepsilon_{i j}$ and $\sigma_{i j}$ are well depth and finite distance at which the interparticle potential is zero between a non-bonded pair of atoms $i$ and $j$ respectively. $r_{i j}$ corresponds to the distance between atoms $i$ and $j$. The material properties and Lennard-Jones potential function parameters for this research are given in the Table 1.

All simulations were performed with a 1 fs integration time step. Data were recorded every 0.005 ns. The cutoff distance for Van der Waals interactions was $12 \AA$.

\section{Molecular Dynamics Model}

Figure 2 shows the MD model and the process steps for the nanoimprinting of gold. The gold substrate had 64512 atoms with dimensions of $10 \mathrm{~nm} \times 10 \mathrm{~nm} \times 5$ $\mathrm{nm}$. The Si mold had 22048 atoms with a tooth depth of $5 \mathrm{~nm}$ and tooth width of $3.8 \mathrm{~nm}$. Periodic boundary conditions were applied in both $\mathrm{x}$ and $\mathrm{y}$ directions. Two layers of atoms at the bottom of the gold substrate were fixed. Figure $2 \mathrm{a}$ depicts the first step of the process in which the temperature of systems was raised to $473 \mathrm{~K}$, which is the recrystallization temperature of the gold. During initial position and temperature ramp-up phase the atoms of gold were in the same position. Figure $2 b$ shows the actual process of imprinting, where the mold was loaded into the substrate at a constant velocity. Steered molecular dynamics was used to impart a velocity of $50 \mathrm{~m} / \mathrm{s}$ to the mold. The mold was held in the substrate for $0.5 \mathrm{~ns}$ to initiate pattern transfer. In this phase, the gold atoms clustered around the mold tooth due to imprinting force. However, the atoms in the rest of the substrate geometry maintain their original position. In this position, the temperature of the system was gradually ramped down to $293 \mathrm{~K}$ for $0.5 \mathrm{~ns}$. On reaching room temperature $(293 \mathrm{~K})$ the system underwent relaxation for $0.5 \mathrm{~ns}$.

Table 1: Material properties and LJ parameters

\begin{tabular}{lrr}
\hline Parameter & Substrate (gold) & Mold (silicon) \\
\hline$\varepsilon, \mathrm{kcal} / \mathrm{mol}$ & -0.0390 & -0.310 \\
$\sigma_{i j}, \AA$ & 3.6900 & 4.270 \\
Melting point, K & 1337.0000 & 1687.000 \\
Density, $\mathrm{kg} / \mathrm{m}^{3}$ & 19300.0000 & 2328.000 \\
Lattice constant, $\AA$ & 4.0788 & 5.431 \\
\hline
\end{tabular}




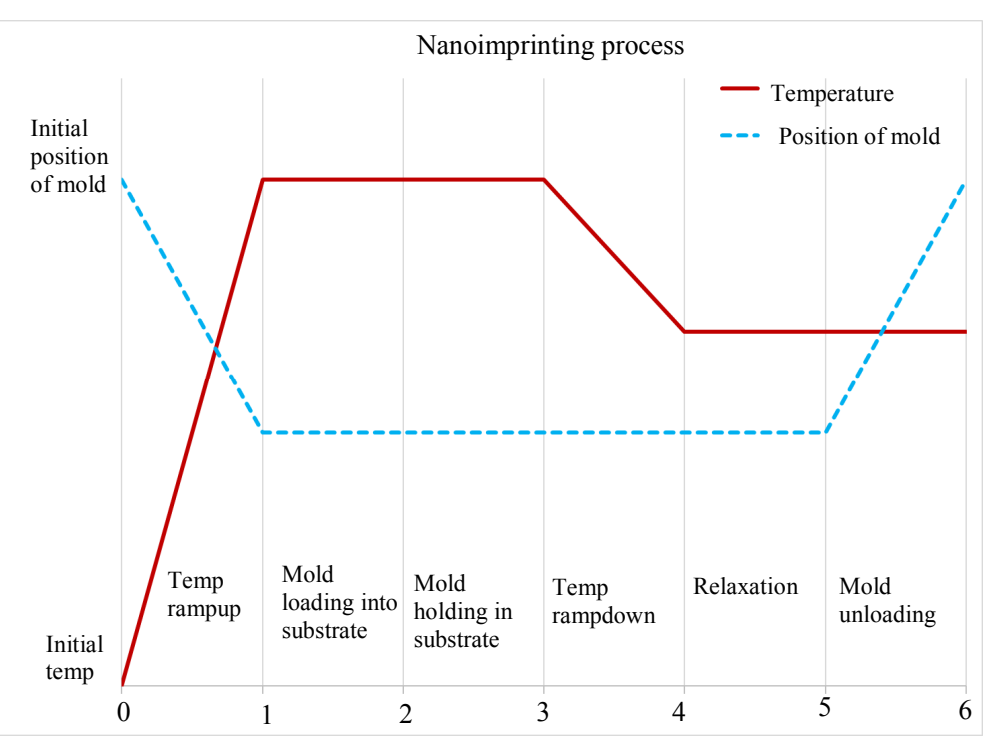

Fig. 1: Phases in the nanoimprint process

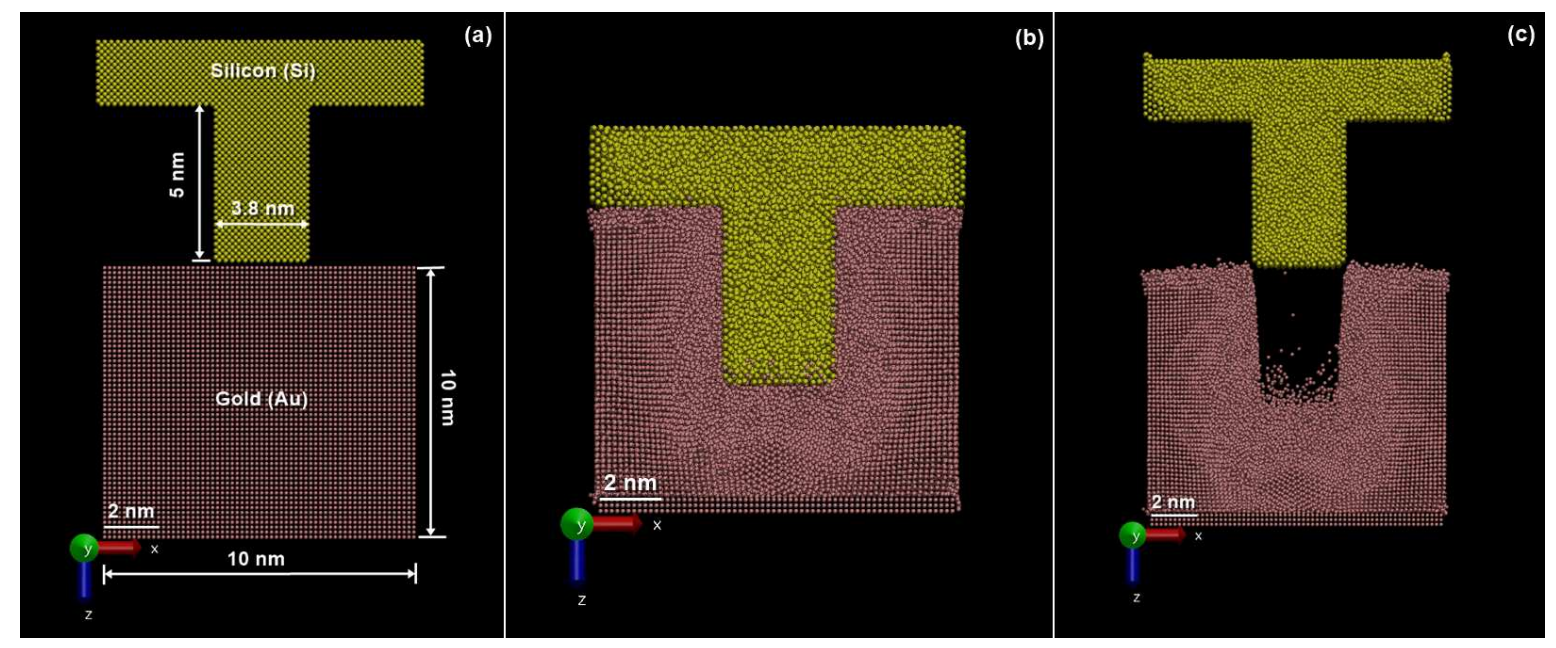

Fig. 2: Phases in nanoimprinting process (a) Initial position and temperature ramp up (b) Mold loading into substrate, mold hold, temperature ramp down and relaxation (c) Mold unloading from the substrate

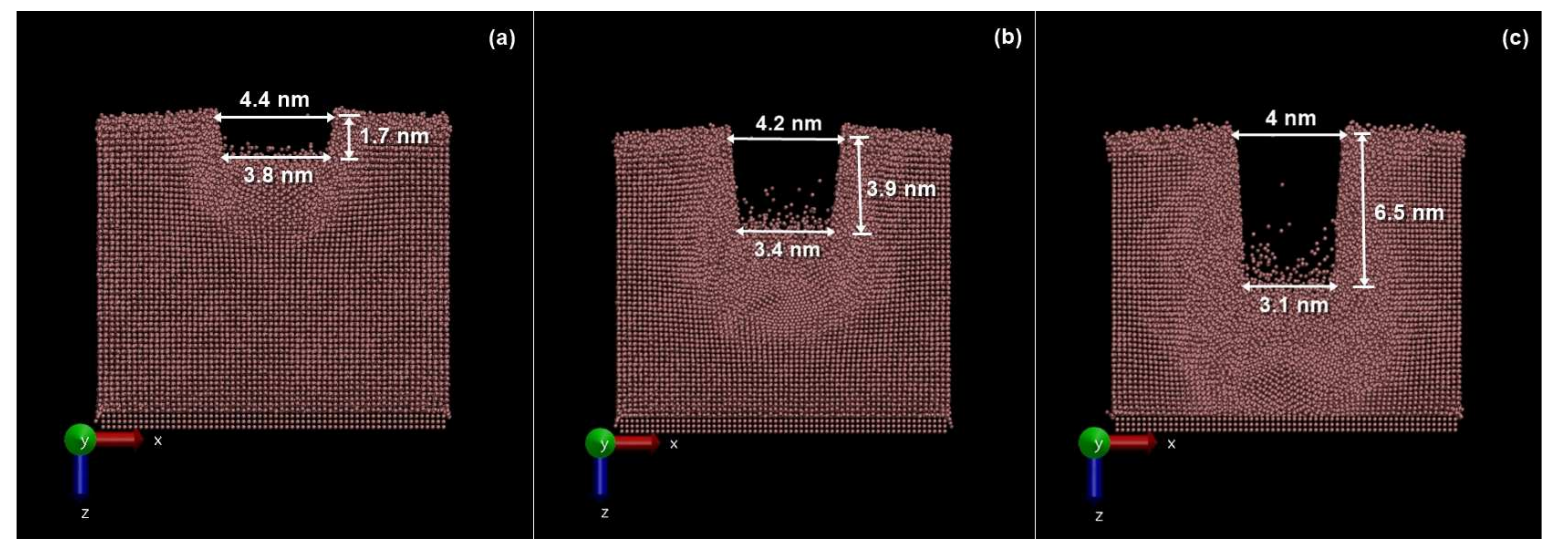

Fig. 3: Substrate after nanoimprinting process (a) 500,000 time steps (b) 1 million time steps (c) 1.5 million time steps 
The plastic deformation and relaxation of the gold substrate occurred during the phases of mold loading, mold holding and temperature ramp down phase in the process. After this phase the mold was unloaded from the gold substrate at a velocity of $50 \mathrm{~m} / \mathrm{s}$. Figure $2 \mathrm{c}$ shows the final deformation of the gold substrate after the nanoimprint process. The gold substrate underwent recrystallization to retain the shape of the mold tooth. A spring back effect was detected on the imprinted pattern when the mold was unloaded from the substrate.

\section{Results and Discussion}

\section{Effect of Imprint Depth on Deformation Geometry and Spring Back Effect}

The silicon mold was imprinted into the gold substrate at different imprint depths namely; 500,000 time steps, 1 million time steps and 1.5 million time steps. Figure 3 depicts the shape of the imprinted substrate at different depths. It was revealed that as the number of steps increase (i.e. as the mold imprints deeper into the substrate) the resultant negative replica shape of mold in the substrate gets narrower. The original width of the mold tooth was $3.8 \mathrm{~nm}$. For a perfect replication of the mold, an imprinted substrate would form a cavity of $3.8 \mathrm{~nm}$ in width. However, due to material spring back phenomenon in metals the imprinted dimension would be lower than $3.8 \mathrm{~nm}$ as evident from Fig. 3. The width of the 500,000 steps imprint at the bottom and top of the imprinted cavity were $3.8 \mathrm{~nm}$ and $4.4 \mathrm{~nm}$, respectively. Whereas the cavity for 1 million steps measured $3.4 \mathrm{~nm}$ and $4.2 \mathrm{~nm}$, respectively. Finally, the imprint cavity for 1.5 million steps measured 3.1 $\mathrm{nm}$ and $4 \mathrm{~nm}$, respectively.

Figure 4 describes the variation in the imprinted cavity for each simulation condition. At the end of mold loading phase, the imprint depths for 500,000 time step, 1 million time step and 1.5 million time step were $1.7 \mathrm{~nm}, 3.9 \mathrm{~nm}$ and $6.5 \mathrm{~nm}$ respectively. The final imprinted depths after unloading the mold for 500,000, 1 million and 1.5 million time steps were $1 \mathrm{~nm}, 3 \mathrm{~nm}$ and $6 \mathrm{~nm}$ respectively. It is important to note that for 1.5 million time step simulation the mold tooth was imprinted beyond its depth (i.e. $5 \mathrm{~nm}$ ) into the substrate. This resulted in an imprinted depth larger $(6 \mathrm{~nm})$ than the original depth $(5 \mathrm{~nm})$ of the mold tooth. Thus, based on both shrinkage in the dimensions along the lateral and depth directions for the imprinted substrate a spring back effect was confirmed. Also, some substrate atoms were dislodged from their imprinted positions due to adhesion energies between the mold and substrate atoms. This was prominently observed in the region of higher densities as compared to the rest of the mold geometry. This was due to the compressive forces subjected by the mold which increased the localized density of atoms in the substrate. Further, the substrate was stabilized by holding the mold in the loaded position at the recrystallization temperature and gradually ramping down the temperature to the room temperature.

\section{Effect of Imprint Depth on Density Profile of Gold Substrate}

The density profile of the substrate for different imprint conditions was evaluated at each phase during the nanoimprinting process. Figures 5, 6 and 7 depicts the change in the density of substrate atoms at different imprint depths. The density of gold at $293 \mathrm{~K}$ is 19.32 $\mathrm{g} / \mathrm{cm}^{3}$. The atomic mass of gold is $196.966 \mathrm{~g} / \mathrm{mol}$. After transforming the density using the Avogadro's constant the atomic density of gold is 0.06 atoms $/ \AA^{3}$.

Figure 5 describes the density profile of gold substrate for 500,000 time step simulation. At initial position and temp ramp-up phase, the density of substrate stayed constant at 0.06 atoms $/ \AA^{3}$ which is a normal density of the gold substrate. As the mold loaded into the substrate the density of substrate atoms increased. When mold reached 500,000 steps (i.e. $1.7 \mathrm{~nm}$ into the substrate), density reached 0.077 atoms $/ \AA^{3}$ which was the highest density. This increase occurred as the gold atoms clustered around the mold tooth. This cluster of gold atoms was seen in the region of actual deformation from $1 \mathrm{~nm}$ to $2 \mathrm{~nm}$. However, the density in the rest of the substrate region remained normal. During the mold holding, temp ramp down and relaxation phases the recrystallization of atoms occurred after the plastic deformation which led to the lowering of the material density. Finally, after mold unloading, the density of the gold substrate remained at 0.067 atoms $/ \AA^{3}$.

Figure 6 represents the density profile of the substrate after 1 million time steps (i.e. $3.9 \mathrm{~nm}$ deep). The highest density was 0.09 atoms $/ \AA^{3}$ at the end of mold loading phase. This increase in density was attributed to the physical deformation of substrate due to the compressive forces subjected by the mold. The density was highest between $3 \mathrm{~nm}$ to $4 \mathrm{~nm}$, which was the final depth of the mold after 1 million time steps. In the remaining phases, the substrate atoms recrystallized resulting in lowering the density to 0.070 atoms $/ \AA^{3}$ in the region of deformation. The density in rest of the substrate was normal.

Figure 7 illustrates the density profile for the 1.5 million time steps (i.e. $6.5 \mathrm{~nm}$ deep) simulation. After the mold loading phase, the density of substrate was 0.094 atoms $/ \AA^{3}$. This was the highest density in the region of deformation from $6 \mathrm{~nm}$ to $7 \mathrm{~nm}$. During the subsequent phases of the process, the density of substrate atoms decreased slowly due to the recrystallization of atoms after the imprinting. At the end of mold unloading phase, the density of substrate atoms remained at 0.075 atoms $/ \AA^{3}$. 


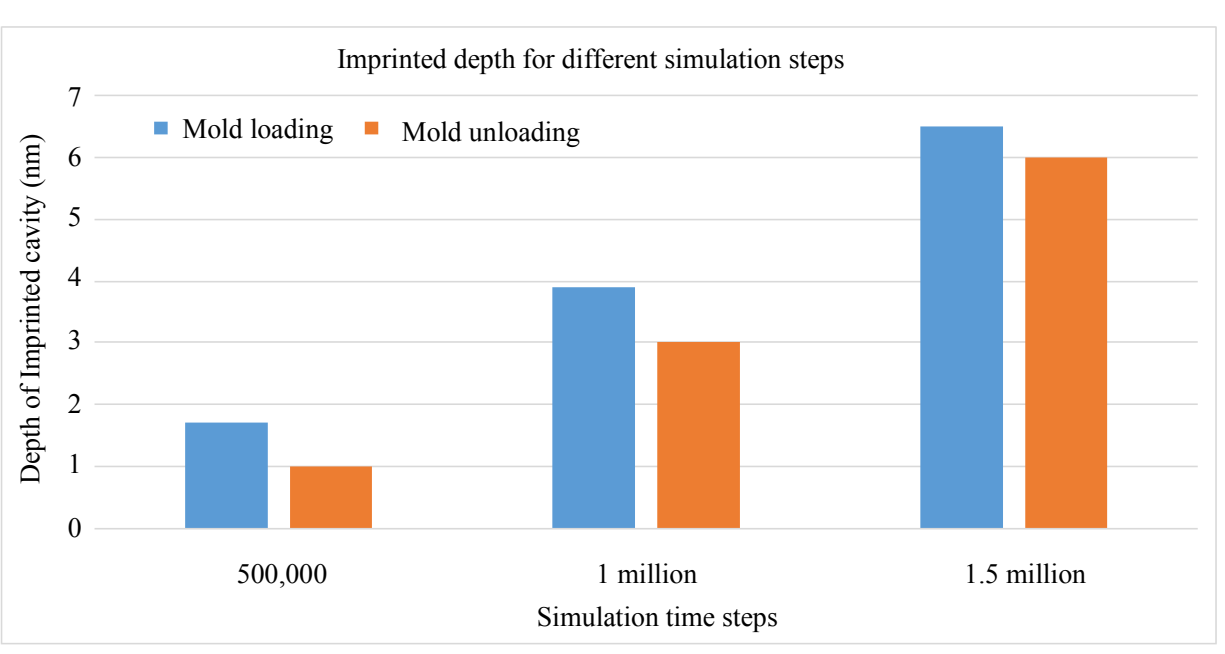

Fig. 4: Imprinted cavity depth vs simulation time steps

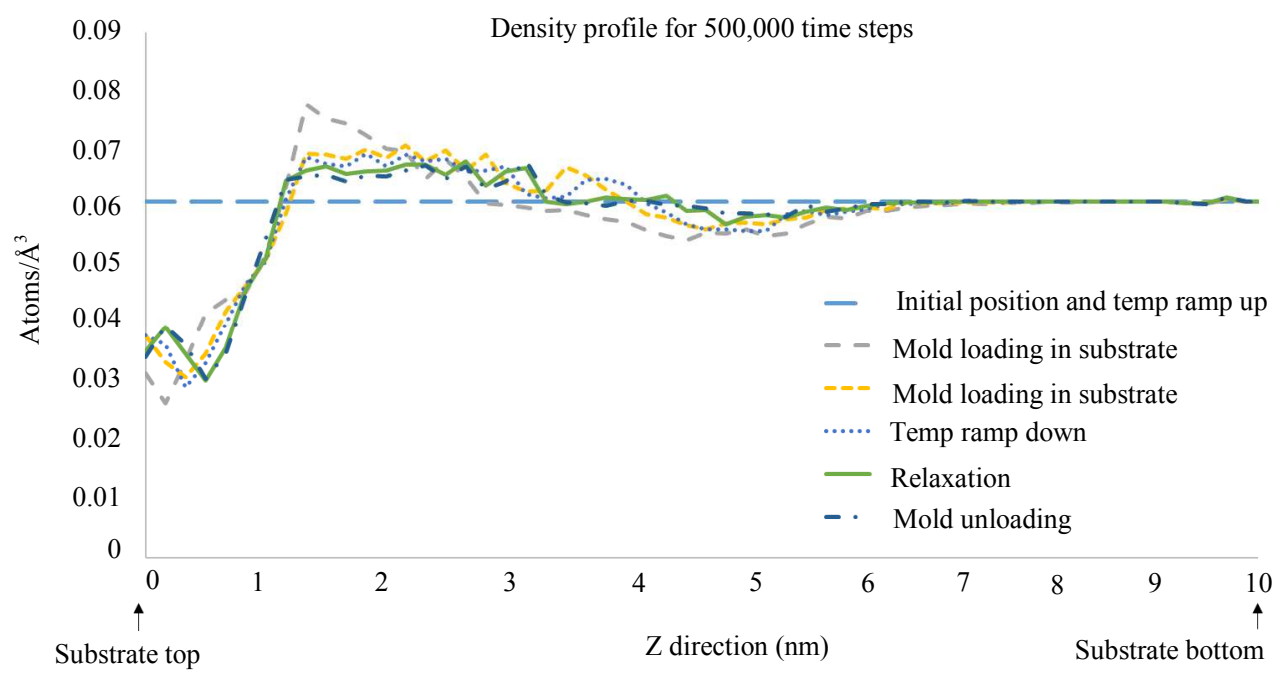

Fig. 5: Density profile of substrate after 500,000 time steps

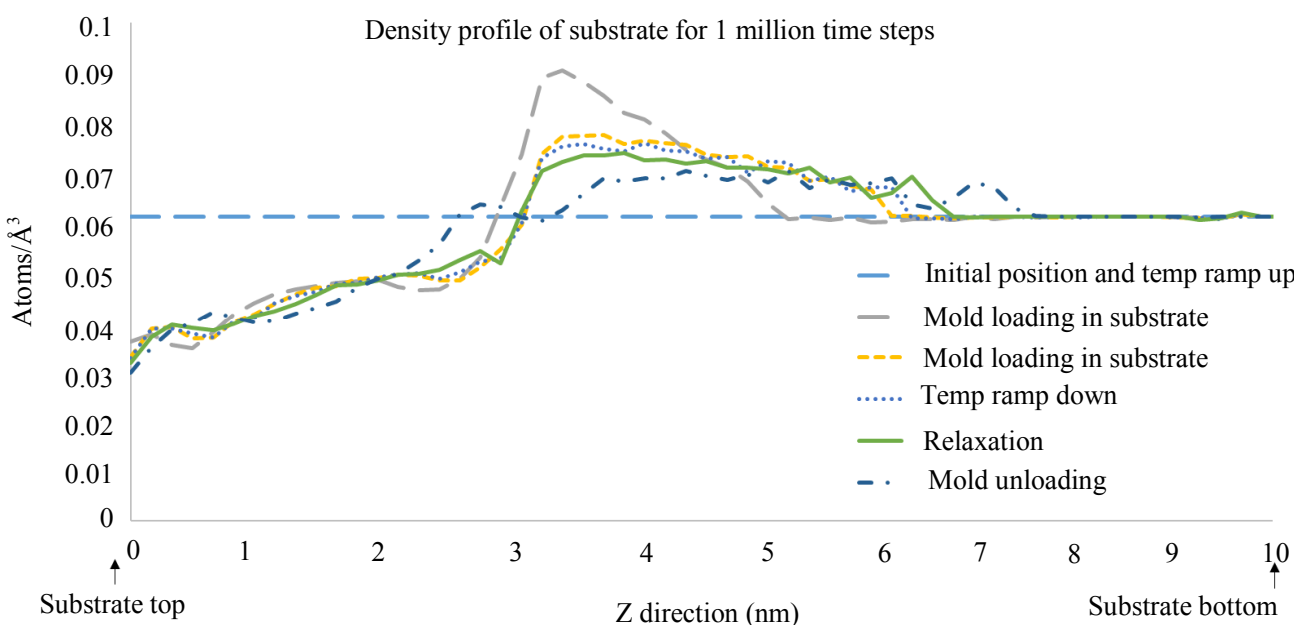

Fig. 6: Density profile of after 1 million time steps 


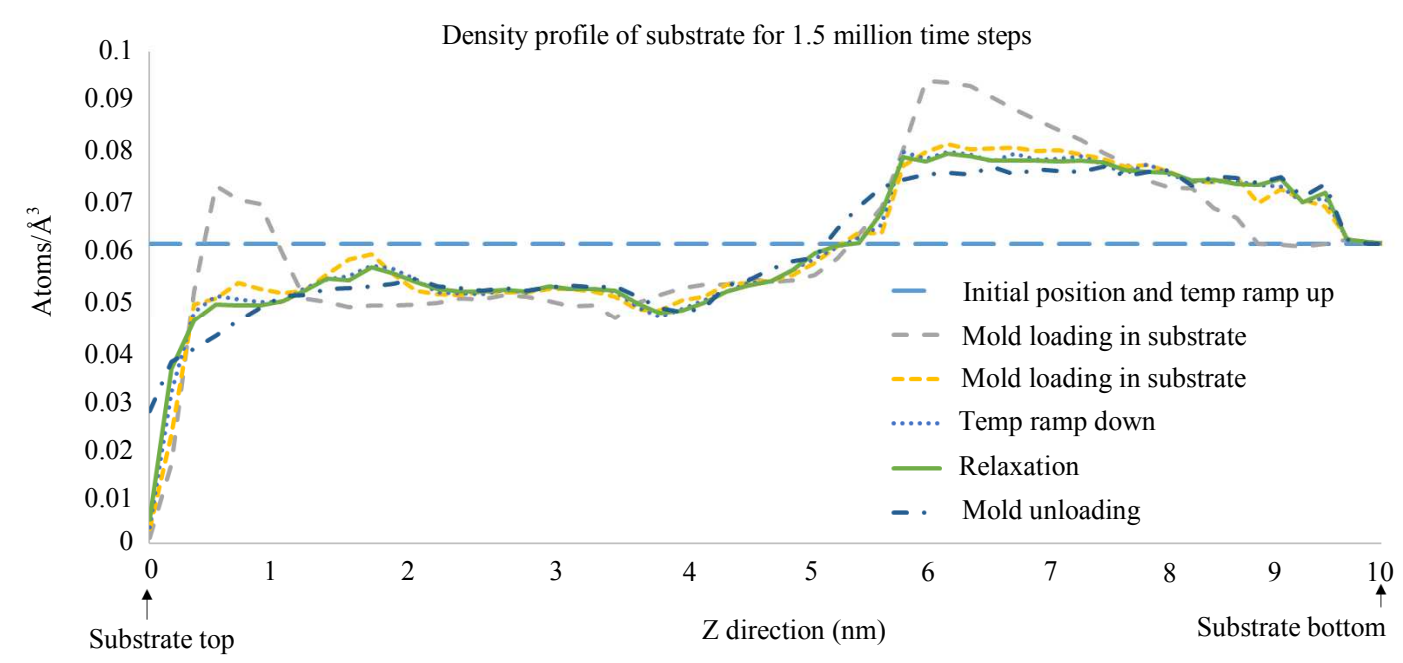

Fig. 7: Density profile of substrate after 1.5 million time steps

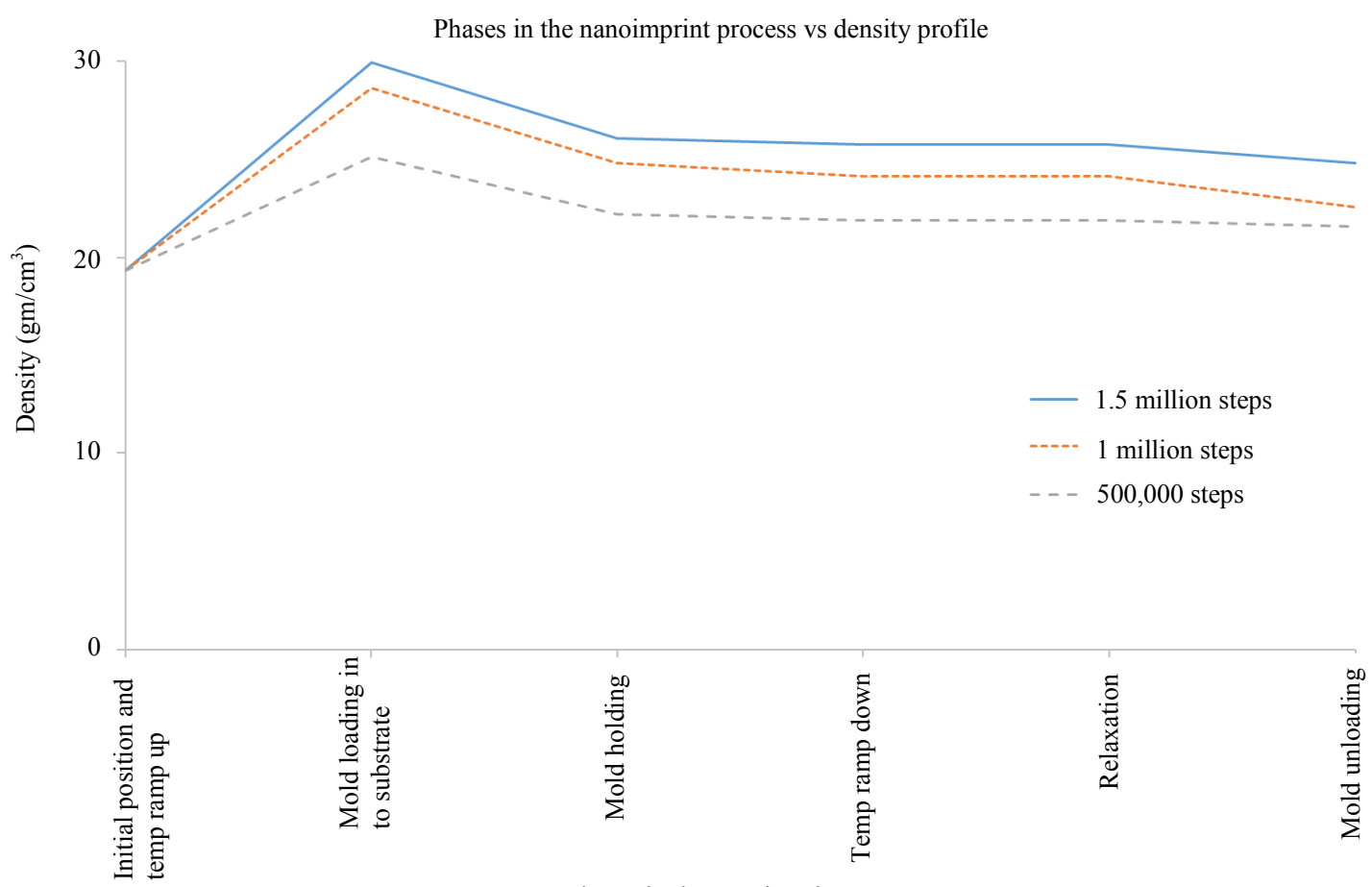

Phases in the nanoimprint process

Fig. 8: Phases in the nanoimprint process vs density profile

Figure 8 shows the variation in the density of substrate at each phase of mold loading for different imprint depths. For the 1.5 million time step simulation the density during the mold loading reached to 29.95 $\mathrm{g} / \mathrm{cm}^{3}$ which was 1.55 times the normal density. Similarly, for 1 million time steps and 500,000 time steps the density during mold loading increased by 1.50 and 1.32 times the normal density, respectively. The MD simulations revealed that as the number of steps (i.e. imprint depth) increased the density of atoms in the substrate increased. In addition, the peak density was attained after the mold loading phase followed by subsequent lowering of density during the recrystallization and unloading phases of the imprinting process. The variations in the density profile were observed for different imprinting depths.

\section{Conclusion}

This paper investigates the physical deformation of the gold substrate by a silicon mold during the nanoimprinting process. The silicon mold was loaded for 
$500,000,1$ million and 1.5 million time steps within the gold substrate. A spring back phenomenon was observed and validated by measuring the imprinted cavity for all the three imprint depths. The simulations with 1.5 million steps had the narrowest width of $3.1 \mathrm{~nm}$ which indicated that as the mold is loaded deep into the substrate the spring back effect was prominent. The density of atoms in the region close to the mold tooth was the highest as compared to the outer regions of the substrate. In addition, it was revealed that the density of atoms in the gold substrate was highest during the mold loading phase of the nanoimprinting process. Variations in the density profile were observed for different imprinting stages. Recrystallization of substrate molecules during mold holding and subsequent cooling phase resulted in lowering of substrate density. Substrate molecules were dislodged from their imprint position during mold unloading due to higher adhesion energies between the $\mathrm{Si}$ mold and $\mathrm{Au}$ substrate. This research lays the foundation for identifying the relationship between mold geometry and imprinted cavity for successful pattern replication.

\section{Acknowledgement}

We would like to thank the US National Science Foundation (NSF CMMI: Award 1663128) for their support.

\section{Author's Contributions}

Abhaysinh Gaikwad and Salil Desai: Performed simulation work and conducted analysis of the research. All authors contributed to the writing of the manuscript.

\section{Ethics}

The authors declare that there are no ethical issues that could arise after the publication of this study.

\section{References}

Chou, S.Y. and P.R. Krauss, 1997. Imprint lithography with sub-10 nm feature size and high throughput. Microelectronic Eng., 35: 237-240.

DOI: $10.1016 / \mathrm{S} 0167-9317(96) 00097-4$.

Chou, S.Y., P.R. Krauss and P.J. Renstrom, 1996. nanoimprint lithography. J. Vacuum Sci. Technol. B: Microelectronics Nanometer Structures Processing Measurement Phenomena, 14: 4129-4133. DOI: $10.1116 / 1.588605$

Cordeiro, J. and S. Desai, 2015. Process parameter studies of molecular dynamics models to control substrate wettability. Proceedings of the International Manufacturing Science Engineering Conference, June 8-12, Manufacturing Engineering Division, Charlotte, North Carolina, USA, pp: 1-7. DOI: $10.1115 / \mathrm{MSEC} 2015-9282$
Cordeiro, J. and S. Desai, 2016. The leidenfrost effect at the nanoscale. J. Micro Nano-Manufacturing, 4: 041-001 DOI: 10.1115/1.4034607

Cordeiro, J. and S. Desai, 2016. The leidenfrost effect at the nanoscale. Proceedings of the 11th International Manufacturing Science Engineering Conference, Jun. 27-Jul. 1, Manufacturing Engineering Division Blacksburg, Virginia, USA, pp: 6.

DOI: $10.1115 /$ MSEC2016-8650

Cordeiro, J. and S. Desai, 2017. The effect of water droplet size, temperature and impingement velocity on gold wettability at the nanoscale. J. Micro NanoManufacturing, 5: 1-8. DOI: 10.1115/1.4036891

Giorgino, T., 2014. Computing 1-D atomic densities in macromolecular simulations: The density profile tool for VMD. Comput. Phys. Commun., 185: 317-322. DOI: 10.1016/j.cpc.2013.08.022

Hsiung, H.Y., H.Y. Chen and C.K. Sung, 2009. Temperature effects on formation of metallic patterns in direct nanoimprint technique-Molecular dynamics simulation and experiment. J. Mater. Process. Technol., 209: 4401-4406. DOI: $10.1016 /$ j.jmatprotec.2008.11.030

Hsu, Q.C., C.D. Wu and T.H. Fang, 2005. Studies on nanoimprint process parameters of copper by molecular dynamics analysis. Comput. Mater. Sci., 34: 314-322. DOI: 10.1016/j.commatsci.2005.01.004

Humphrey, W., A. Dalke and K. Schulten, 1996. VMD: visual molecular dynamics. J. Molecular Graphics, 14: 33-38. DOI: 10.1016/0263-7855(96)00018-5

Jiao, S., W. Tu, P. Zhang, W. Zhang and L. Qin et al., 2018. Atomistic insights into the prismatic dislocation loop on $\mathrm{Al}$ (100) during nanoindentation investigated by molecular dynamics. Comput. Mater. Sci., 143: 384-390. DOI: 10.1016/j.commatsci.2017.11.031

Kang, J.H., K.S. Kim and K.W. Kim, 2010. Molecular dynamics study on the effects of stamp shape, adhesive energy and temperature on the nanoimprint lithography process. Applied Surface Sci., 257: 1562-1572.

DOI: 10.1016/j.apsusc.2010.08.096

Kwon, B. and J.H. Kim, 2016. Importance of molds for nanoimprint lithography: Hard, soft and hybrid molds. J. Nanosci., 2016: 1-12. DOI: 10.1155/2016/6571297

Lin, Y.C. and Z.I. Yu, 2017. Molecular dynamics simulation of nanoimprinting effects of silver thin films with impurities defects on copper substrate. Proceedings of the International Conference Applied System Innovation, May 13-17, IEEE Xplore Press, Sapporo, Japan, pp: 1520-1523. DOI: $10.1109 /$ ICASI.2017.7988213

Pease, R.F. and S.Y. Chou, 2008. Lithography and other patterning techniques for future electronics. Proc. I., 96: 248-270. DOI: 10.1109/JPROC.2007.911853 
Phillips, J.C., R. Braun, W. Wang, J. Gumbart and E. Tajkhorshid et al., 2005. Scalable molecular dynamics with NAMD. J. Computational Chem., 26: 1781-1802. DOI: 10.1002/jcc.20289

Quang-Cherng, H., W. Chen-Da and F. Te-Hua, 2004. Deformation mechanism and punch taper effects on nanoimprint process by molecular dynamics. Japanese J. Applied Phys.

Vigneswaran, N., F. Samsuri, B. Ranganathan and Padmapriya, 2014. Recent advances in nano patterning and nano imprint lithography for biological applications. Procedia Eng., 97: 1387-1398.

DOI: 10.1016/j.proeng.2014.12.420

Wong, T.I., S. Han, L. Wu, Y. Wang and J. Deng et al., 2013. High throughput and high yield nanofabrication of precisely designed gold nanohole arrays for fluorescence enhanced detection of biomarkers. Lab Chip, 13: 2405-2413.

DOI: $10.1039 /$ C3LC41396A
Wu, C.D. and C.J. Hou, 2018. Molecular dynamics analysis of plastic deformation and mechanics of imprinted metallic glass films. Comput. Mater. Sci., 144: $248-255$

DOI: 10.1016/j.commatsci.2017.12.042

Yang, S., S. Yu and M. Cho, 2014. Influence of mold and substrate material combinations on nanoimprint lithography process: MD simulation approach. Applied Surface Sci., 301: 189-198. DOI: 10.1016/j.apsusc.2014.02.037

Yasuda, M., K. Tada and Y. Hirai, 2010. Molecular Dynamics Study on Mold and Pattern Breakages in Nanoimprint Lithography. 1st Edn., INTECH Open Access Publisher, ISBN -10: 9533070641.

Zhang, J., Y. Zhang, N.A. Mara, J. Lou and L. Nicola, 2014. Direct nanoimprinting of single crystalline gold: Experiments and dislocation dynamics simulations. Applied Surface Sci., 290: 301-307. DOI: 10.1016/j.apsusc.2013.11.072 